\title{
Connecting physics to the economy
}

\author{
How important is physics to the economies of European countries?
}

The contribution of physics-based industries to European economies is large and growing significantly, according to a recent report published by the European Physical Society (EPS) entitled "The importance of physics to the economies of Europe". The EPS commissioned the Centre for Economics and Business Research to conduct this economic analysis in 2012. Based on statistics in the public domain through Eurostat, the analysis in the report is both quantitative and, as much as possible, unbiased. It covers 29 European countries (hereafter Europe) - the EU27 countries, plus Norway and Switzerland - over the period 2007-2010 (2010 being the most recent year for which official data are available for all 29 countries).

Remarkably, the report shows that the physics-based industrial sector generated a turnover of more than $€ 3.4$ trillion each year in the period 2007-2010. Specifically, the sector generated $€ 4.0$ trillion in 2008 , $€ 3.48$ trillion in 2009 (the year of the global economic downturn) and $€ 3.8$ trillion in 2010 . These values typically represent over $15 \%$ of the total turnover of Europe's business economy and exceed the combined contributions of the entire retail sector. Of the 29 European countries, Germany, France and the UK were the three largest contributors in 2010 to the physics-related turnover, accounting for $25.3 \%, 12.4 \%$ and $11.9 \%$ of this turnover, respectively.

The importance of physics is also reflected in the gross value added (GVA) a measure of the value generated in the production of goods by particular sectors of the economy (thus analogous to the gross domestic product). With the exception of 2009, the GVA of physics-based industries exceeded $€ 1.25$ trillion each year in the period 2007-2010. This amount corresponds to about $11 \%$ of the total GVA of the EU27 economies, which is a greater proportion than that of the construction, financial or retail sectors.

With these impressive statistics for the turnover and the GVA, it is not surprising to learn that physics-based industries generated many jobs in Europe. Each year in the period 2007-2010, over 15.3 million people were employed in the physics-based sector, which accounts for over $13 \%$ of the total employment in Europe. Furthermore, for every physics-related job created,

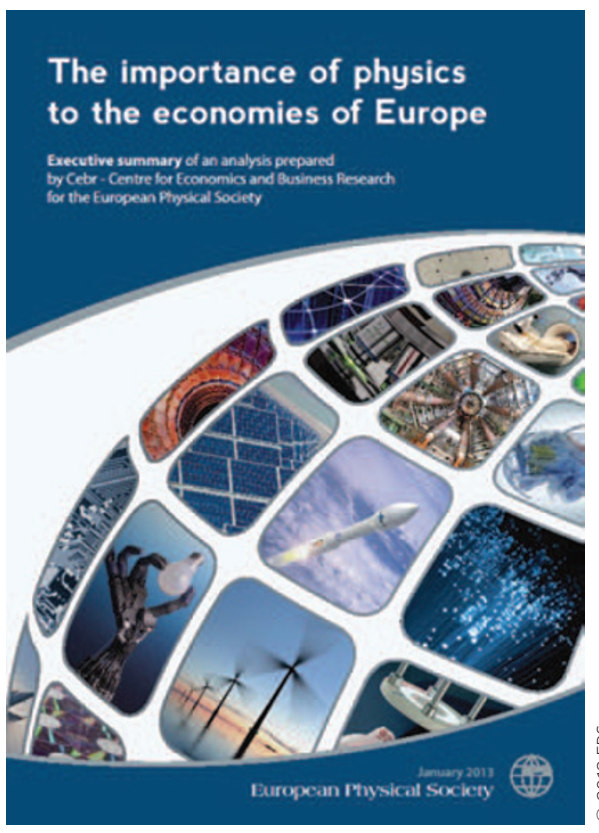

particularly relevant in the context of austere budgets to demonstrate the importance and the impact of physics research to innovation, growth and development. En passant, the report also defines the discipline of physics and provides the contours for the identification of the so-called physics-based industry." says Luisa Cifarelli, the President of the EPS.

Cifarelli also added that by demonstrating the impact of physics on the European economy, the EPS has highlighted the need to continue supporting physics research and education, both at the European and national levels. Cifarelli believes that the EPS should actively disseminate this message by initiating similar surveys across the 41 European countries that host its member societies, as well as regularly repeating the Europeanwide survey.

When asked what governments can do to help, Cifarelli urges them to "recognize that funding scientific research and education is an investment for the future. Not only with regards to the economy, but also with regards to general technological development in a wide range of diverse areas, such as health, energy, communications and so on. This is crucial to achieve steady progress and long-lasting welfare."

She also urges the physics community to actively use the information in the report to promote the vital role of physics in culture as well as the economy. "The physics community now has a powerful tool to show that physics counts," Cifarelli insists.

The Commentary on page 338 of this issue strikes a similar note. John Dudley from the University of FrancheComté emphasizes the benefits of basic research and the need to champion them aggressively. Governments are demanding more value for money from scientists, thus putting fundamental research under growing pressure.

"Fundamental discoveries in physics and other disciplines are incorporated in many of the technologies that we now take for granted, and they drive economic growth both directly and indirectly," Dudley points out. He concludes by remarking, "It is essential that scientists know how to defend [the benefits of fundamental research] effectively." 